\title{
Comparaison des activités des isotopes de l'uranium et du radium dans quelques échantillons d'eau de puits et de sources thermales au Maroc \\ Comparison of uranium and radium isotopes activities in some well and thermal springs samples in Morroco
}

\author{
O. K. Hakam, A. Choukri, J. L. Reyss et M. Lferde
}

Volume 13, numéro 2, 2000

URI : https://id.erudit.org/iderudit/705390ar

DOI : https://doi.org/10.7202/705390ar

\section{Aller au sommaire du numéro}

\section{Éditeur(s)}

Université du Québec - INRS-Eau, Terre et Environnement (INRS-ETE)

ISSN

0992-7158 (imprimé)

1718-8598 (numérique)

Découvrir la revue

Citer cet article

Hakam, O. K., Choukri, A., Reyss, J. L. \& Lferde, M. (2000). Comparaison des activités des isotopes de l'uranium et du radium dans quelques échantillons d'eau de puits et de sources thermales au Maroc. Revue des sciences de l'eau / Journal of Water Science, 13(2), 185-192. https://doi.org/10.7202/705390ar
Résumé de l'article

Les activités des isotopes de l'uranium et du radium $\left({ }^{234} \mathrm{U},{ }^{238} \mathrm{U},{ }^{226} \mathrm{Ra},{ }^{228} \mathrm{Ra}\right)$ ainsi que les rapports d'activité $\left({ }^{234} \mathrm{U} /{ }^{238} \mathrm{U},{ }^{226} \mathrm{Ra} /{ }^{238} \mathrm{U},{ }^{228} \mathrm{Ra} /{ }^{226} \mathrm{Ra}\right)$ ont été mesurés, pour la première fois au Maroc, dans 15 échantillons d'eau de puits et 12 échantillons d'eau de sources thermales. Les résultats obtenus montrent que, contrairement aux eaux de puits, les eaux des sources thermales présentent des activités de ${ }^{238} \mathrm{U}$ relativement faibles et des activités de ${ }^{226} \mathrm{Ra}$ et des rapports ${ }^{234} \mathrm{U} /{ }^{238} \mathrm{U}$ élevés. Les activités de l'uranium et du radium mesurées sont comparables à celles que l'on trouve habituellement dans d'autres régions non polluées du monde, elles sont inférieures aux limites maximales admissibles ce qui ne pose aucun risque pour la santé publique au Maroc. 


\section{Comparaison des activités des isotopes de l'uranium et du radium dans quelques échantillons d'eau de puits et de sources thermales au Maroc}

\section{Comparison of uranium and radium isotopes activities in some well and thermal springs samples in Morroco}

\section{K. HAKAM ${ }^{*}, 1,3$, A. CHOUKRI ${ }^{1,}{ }^{*}$, J.-L. REYSS ${ }^{2}$, M. LFERDE ${ }^{3}$}

Reçu le 21 janvier 1999, accepté le 26 juin 2000**.

\section{SUMMARY}

Activities and activity ratios of uranium and radium isotopes $\left({ }^{234} \mathrm{U},{ }^{238} \mathrm{U}\right.$, ${ }^{226} \mathrm{Ra},{ }^{228} \mathrm{Ra},{ }^{234} \mathrm{U} /{ }^{238} \mathrm{U},{ }^{226} \mathrm{Ra} /{ }^{238} \mathrm{U},{ }^{228} \mathrm{Ra} /{ }^{226} \mathrm{Ra}$ ) have been determined, for the first time in Morocco, for 15 well water samples and 12 spring water samples. The obtained results show that, unlike well waters, the thermal spring waters present relatively low ${ }^{238} \mathrm{U}$ activities and elevated ${ }^{226} \mathrm{Ra}$ activities and ${ }^{234} \mathrm{U} /{ }^{238} \mathrm{U}$ activity ratios. Uranium and radium activities are similar to those published for other non polluting regions of the world, they are inferior to the Maximum Contaminant Levels and don't present any risk for public health in Morocco.

Key-words: thermal springs, well waters, natural waters, drinking waters, uranium, radium, natural radioactivity.

RESUMÉ

Les activités des isotopes de l'uranium et du radium $\left({ }^{234} \mathrm{U},{ }^{238} \mathrm{U},{ }^{226} \mathrm{Ra},{ }^{228} \mathrm{Ra}\right)$ ainsi que les rapports d'activité $\left.{ }^{234} \mathrm{U} /{ }^{238} \mathrm{U},{ }^{226} \mathrm{Ra} /{ }^{238} \mathrm{U},{ }^{228} \mathrm{Ra} /{ }^{226} \mathrm{Ra}\right)$ ont été mesurés, pour la première fois au Maroc, dans 15 échantillons d'eau de puits et 12 échantillons d'eau de sources thermales. Les résultats obtenus montrent que, contrairement aux eaux de puits, les eaux des sources thermales présentent des activités de ${ }^{238} \mathrm{U}$ relativement faibles et des activités de ${ }^{226}$ Ra et des rapports ${ }^{234} U{ }^{238} \mathrm{U}$ élevés. Les activités de l'uranium et du radium mesurées

1. Laboratoire des faibles radioactivités et de l'environnement, Université Ibn Tofail, Faculté des sciences, Département de physique, 14000 , Kénitra, Maroc.

2. Laboratoire des sciences du climat et de l'environnement, Laboratoire mixte CNRS-CEA, 91198 , Domaine du CNRS, avenue de la Terrasse, Gif-sur-Yvette cedex, France.

3. Laboratoire de physique nucléaire, Université Mohammed V, Faculté des sciences, Département de physique, Avenue Ibn, Battouta BP 1014, Rabat, Maroc.

* Correspondance. E-mail : okhakam@yahoo.com ou choukrimajid@yahoo.com

** Les commentaires seront reçus jusqu'au 15 mai 2001. 
sont comparables à celles que l'on trouve habituellement dans d'autres régions non polluées du monde, elles sont inférieures aux limites maximales admissibles ce qui ne pose aucun risque pour la santé publique au Maroc.

Mots clés : sources thermales, eaux de puits, eaux naturelles, eaux potables, uranium, radium, radioactivité naturelle.

\section{1 - INTRODUCTION}

Les puits et les sources constituent actuellement deux sources principales d'eau potable au Maroc. Certaines eaux minérales de sources thermales sont commercialisées ou utilisées comme bains thérapeutiques pour guérir certaines maladies de peau. Les concentrations en éléments radioactifs naturels dans les eaux profondes peuvent être reliées aux conditions physicochimiques de l'eau et à son environnement géologique. Cette radioactivité est essentiellement due à l'uranium, au thorium ainsi qu'à leurs descendants, dont l'eau s'est chargée lors de son passage au travers des roches plutoniques ou métamorphiques profondes. Par exemple, les sources les plus radioactives sont fréquemment les sources carbo-gazeuses ou chlorurées dont les caractéristiques chimiques favorisent la solubilisation des éléments lourds comme l'uranium et le radium.

La radioactivité naturelle dans les eaux souterraines varie beaucoup, des concentrations élevées des radioéléments naturels ont été trouvées, d'après plusieurs travaux (ANDREWS et KAY (1983), ASIKAINEN et KAHOLS (1979), FRANZ (1989)), dans des eaux minérales et des puits. La connaissance des concentrations de l'uranium et du radium dans les eaux potables est importante parce qu'une fraction de l'uranium et du radium absorbés est déposée dans le corps humain introduisant ainsi une dose radioactive interne équivalente.

Le but de ce travail est de comparer les activités des isotopes de l'uranium $\left({ }^{234} \mathrm{U},{ }^{238} \mathrm{U}\right)$ et du radium $\left({ }^{226} \mathrm{Ra},{ }^{228} \mathrm{Ra}\right)$ ainsi que les rapports d'activité $\left({ }^{234} \mathrm{U} /{ }^{238} \mathrm{U},{ }^{226} \mathrm{Ra} /{ }^{238} \mathrm{U},{ }^{228} \mathrm{Ra} /{ }^{226} \mathrm{Ra}\right)$ dans des échantillons d'eau de puits et de sources thermales et voir si la teneur en uranium ou en radium dans ces échantillons présente un risque pour l'hygiène publique. La figure 1 illustre la carte du Maroc avec les sites d'échantillonnage.

\section{2 - PARTIE EXPÉRIMENTALE}

La procédure de mesure, de comptage et de calcul utilisée est identique à celle décrite par SCHMIDT and REYSS (1996) pour la spectrométrie gamma et à celle décrite par CHOUKRI et al. (1994) pour la spectrométrie alpha.

La technique utilisée consiste à prélever, dans des bidons en plastique, 5 litres d'eau pour une analyse unique de l'uranium, et 20 litres d'eau pour une analyse double de l'uranium et du radium. Après filtration sur des membranes 


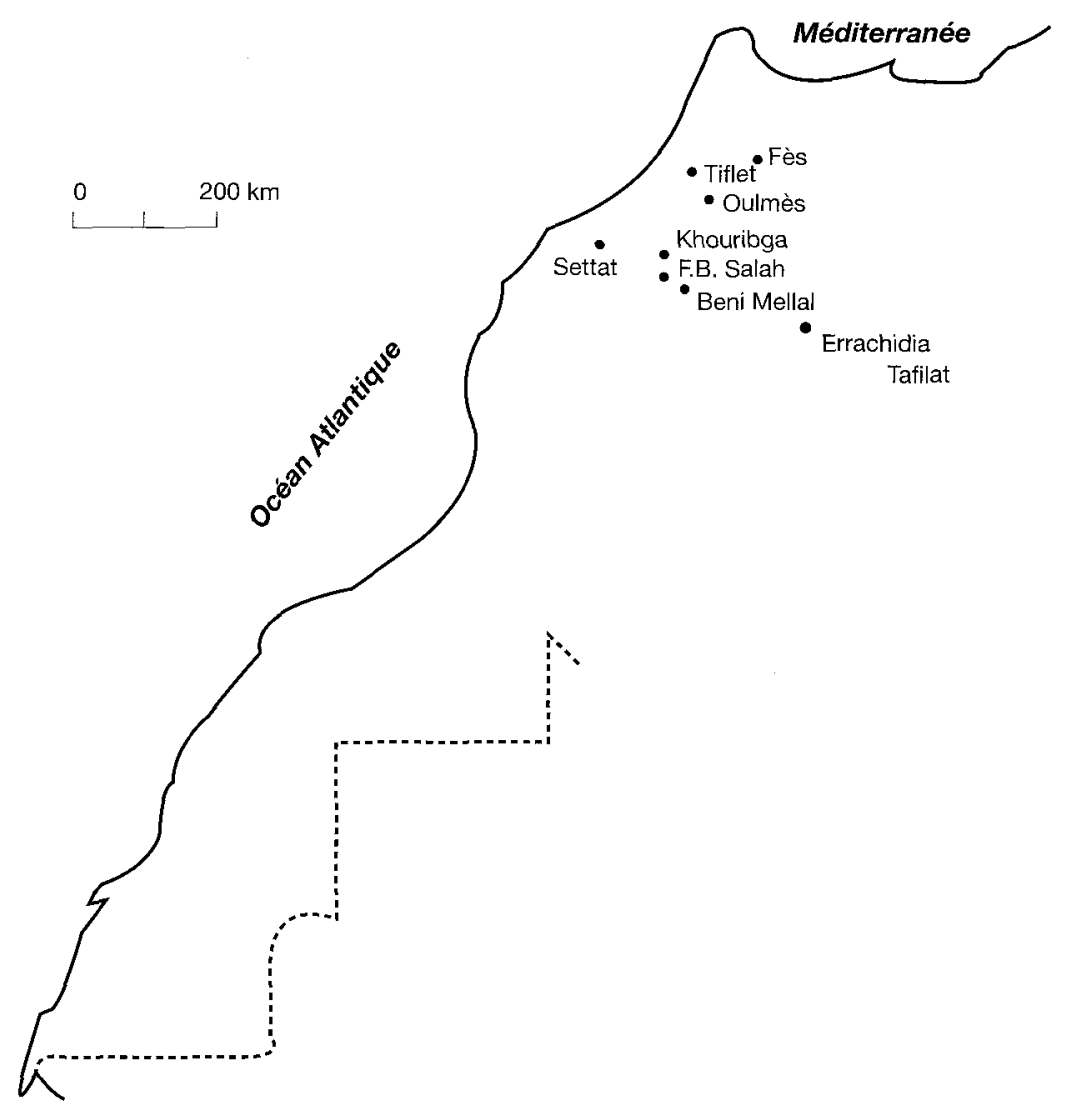

Figure 1 Carte du Maroc avec localisation des sites d'échantillonnage.

Morocco mape with sampling localities.

de marque Millipore de porosité $0,4 \mu \mathrm{m}$ et de diamètre $147 \mathrm{~mm}$, l'échantillon d'eau est acidifié à $\mathrm{pH} 2$ à 3 avec $\mathrm{HNO}_{3}$ avant de rajouter une solution contenant des quantités connues des traceurs $\left({ }^{232} \mathrm{U}\right.$ et $\left.{ }^{228} \mathrm{Th}\right)$ et du fer (50 à $80 \mathrm{mg}$ ), et une solution contenant 1 gramme de chlorure de baryum $\left(\mathrm{BaCl}_{2}\right)$. Les isotopes de l'uranium et du thorium sont coprécipités avec les hydroxydes de fer formés par l'ajout de l'ammoniaque à $\mathrm{pH}=7$ dans le milieu avant de précipiter le radium avec $\mathrm{BaSO}_{4}$ formé par l'ajout de $\mathrm{H}_{2} \mathrm{SO}_{4}$. Le précipité de sulfate de baryum et des hydroxydes de fer sont récupérés ensemble par décantation et filtration. L'uranium et le thorium sont dissous avec les hydroxydes par l'ajout d'acide concentré, tandis que le radium est retenu dans le résidu de sulfate de baryum qui est insoluble dans le milieu acide. Ce résidu est lavé, séché et pesé avant d'être scellé dans un tube convenable pour le comptage dans le détecteur germanium puits à très bas bruit de fond. Le comptage est fait après 20 jours de scellage, temps nécessaire pour que l'équilibre soit établi entre les isotopes du radium et leurs descendants. Les activités des isotopes du radium sont mesurées à l'aide de ses descendants émetteurs gamma de courtes périodes $\left({ }^{214} \mathrm{~Pb}\right.$ et ${ }^{214} \mathrm{Bi}$ pour ${ }^{226} \mathrm{Ra}$ et ${ }^{228} \mathrm{Ac}$ pour $\left.{ }^{228} \mathrm{Ra}\right)$. Elles sont corrigées $\mathrm{du}$ 
rendement de précipitation du sulfate de baryum en tenant compte du facteur de correction du poids $(1,16)$ due à la formation de $\mathrm{BaSO}_{4}$ à partir de $\mathrm{BaCl}_{2}$. Les standards gamma utilisés pour l'intercalibration sont N.B.S (National Bureau of Standard USA) 4363 et 4350 B, et AIEA 375.

La détermination des activités des isotopes de l'uranium $\left({ }^{238} \mathrm{U}\right.$ et $\left.{ }^{234} \mathrm{U}\right)$ est effectuée par spectrométrie alpha en utilisant, soit un détecteur à chambre de gaz, soit un détecteur à barrière de surface. Elle est précédée d'une séparation chimique poussée sur résine échangeuse d'ions selon la procédure chimique décrite par CHOUKRI et al. (1994).

\section{3 - RÉSULTATS ET DISCUSSION}

Les activités des isotopes de l'uranium et du radium $\left({ }^{238} \mathrm{U},{ }^{226} \mathrm{Ra}\right)$ ainsi que les rapports d'activités $\left({ }^{234} \mathrm{U} /{ }^{238} \mathrm{U},{ }^{226} \mathrm{Ra} /{ }^{238} \mathrm{U},{ }^{228} \mathrm{Ra} /{ }^{226} \mathrm{Ra}\right)$ obtenus pour 15 échantillons d'eau de puits et 12 échantillons d'eau de sources thermales, sont donnés dans le tableau 1.

L'activité de ${ }^{238} \mathrm{U}$ varie, dans l'eau de puits, entre 4,5 et $308,4 \mathrm{mBq} / \mathrm{l}$ avec une valeur moyenne de $66 \mathrm{mBq} / \mathrm{l}$ environ, et dans l'eau de sources, entre 0,6 et $17,9 \mathrm{mBq} / \mathrm{l}$ avec une valeur moyenne de $7,7 \mathrm{mBq} / \mathrm{l}$. Les activités du radium mesurées dans 7 puits varient, de 1 à $25 \mathrm{mBq} / \mathrm{l}$ avec une valeur moyenne de $9,8 \mathrm{mBq} / \mathrm{l}$, tandis que celles mesurées dans 9 sources thermales varient entre 9,1 et $3696 \mathrm{mBq} / \mathrm{l}$ avec une valeur moyenne supérieure à $590 \mathrm{mBq} / \mathrm{l}$. L'activité du ${ }^{226} \mathrm{Ra}$, la plus élevée, a été mesurée dans la source de My. Yacoub située dans la région de la ville de Fès connue par sa richesse en sources thermales. L'eau de cette source, non potable à cause de sa richesse en sels dissous, est utilisée comme bain thérapeutique pour guérir certaines maladies de peau. Une comparaison des activités de ${ }^{238} \mathrm{U}$ et du ${ }^{226} \mathrm{Ra}$ dans les eaux de puits et de sources montrent que l'eau de puits est relativement plus riche en uranium et celle des sources est relativement plus riche en radium et que les concentrations en radium ne sont pas nécessairement liées à celles de l'uranium. En effet, les concentrations de l'uranium et du radium sont affectées par la température et l'état rédox de l'eau. Les hautes températures tendent à décroître la concentration de l'uranium et à augmenter celle du radium. La diminution de la concentration de l'uranium résulte de la régression de la stabilité des complexes de carbonates d'uranyle alors que l'augmentation de celle du radium résulte de la réduction des coefficients d'adsorption des ions divalents du radium. Dans des conditions oxydantes, l'uranium est très soluble principalement sous la forme de complexes carbonatés $U(V I)$. Dans des conditions réductrices, l'uranium est insoluble sous la forme d'oxydes-U (IV) (TOULHOAT et al., 1988). La présence du radium qui a un comportement voisin de celui du baryum est limitée en solution par la présence de sulfates. En milieu réducteur tel que celui des eaux géothermiques, la disparition des sulfates permet des concentrations plus importantes en radium.

Le déséquilibre radioactif entre ${ }^{238} U$ et son descendant ${ }^{234} U$ dans les eaux naturelles a été bien étudié depuis sa découverte. Les rapports d'activité ${ }^{234} \mathrm{U} /{ }^{238} \mathrm{U}$, mesurés dans les eaux naturelles, sont souvent supérieurs à 1 
Tableau 1 Activités et rapports d'activité des isotopes de l'uranium et du radium dans les échantillons analysés d'eau de puits et de sources thermales.

Table 1 Activities and activity ratios of uranium and radium radio-isotopes in analyzed well and thermal spring water samples.

\begin{tabular}{|c|c|c|c|c|c|c|c|}
\hline$n^{\circ}$ & échantillon & région & ${ }^{238} \mathrm{U}(\mathrm{mBq} / \mathrm{l})$ & ${ }^{234} \mathrm{U} /{ }^{238} \mathrm{U}$ & ${ }^{226} \mathrm{Ra}(\mathrm{mBq} / \mathrm{l})$ & ${ }^{228} \mathrm{Ra} /{ }^{226} \mathrm{Ra}$ & ${ }^{226} \mathrm{Ra} /{ }^{238} \mathrm{U}$ \\
\hline \multicolumn{8}{|c|}{ Puits } \\
\hline 1 & Settat & Settat & $164,8 \pm 26,8$ & $1,01 \pm 0,04$ & $10,8 \pm 0,5$ & $1,59 \pm 0,14$ & $0,07 \pm 0,01$ \\
\hline 2 & Beni Yakhlef & Khouribga & $4,5 \pm 0,3$ & $0,98 \pm 0,12$ & $5,1 \pm 0,3$ & $0,20 \pm 0,04$ & $1,14 \pm 0,10$ \\
\hline 3 & Ourghida & Khouribga & $22,5 \pm 1,8$ & $1,08 \pm 0,05$ & $25,0 \pm 1,0$ & $0,20 \pm 0,04$ & $1,11 \pm 0,10$ \\
\hline 4 & Lahmina & Khouribga & $26,2 \pm 2,0$ & $1,16 \pm 0,04$ & $15,1 \pm 1,4$ & $0,04 \pm 0,03$ & $0,58 \pm 0,07$ \\
\hline 5 & Ouled Sasi & Khouribga & $63,9 \pm 7,9$ & $0,80 \pm 0,06$ & & & \\
\hline 6 & Ouled Azouz & Khouribga & $62,8 \pm 5,1$ & $0,88 \pm 0,04$ & & & \\
\hline 7 & $1 \mathrm{~km}$ extr. Phos. & Khouribga & $308,4 \pm 27,5$ & $0,79 \pm 0,02$ & & & \\
\hline 8 & Jorf Mellha & Tiflet & $16,8 \pm 1,6$ & $1,45 \pm 0,11$ & & & \\
\hline 9 & Tiflet & Tiflet & $56,7 \pm 4,5$ & $2,83 \pm 0,12$ & & & \\
\hline 10 & Beni Mellal & Beni Mellal & $6,3 \pm 0,5$ & $2,11 \pm 0,13$ & $1,0 \pm 0,2$ & $1,20 \pm 0,38$ & $0,16 \pm 0,03$ \\
\hline 11 & Ouled Réguia & F.B. Salah & $60,6 \pm 4,1$ & $1,01 \pm 0,02$ & $6,3 \pm 0,3$ & $0,76 \pm 0,09$ & $0,10 \pm 0,01$ \\
\hline 12 & Béni-Amir & F.B. Salah & $45,5 \pm 3,8$ & $0,97 \pm 0,03$ & $5,4 \pm 0,4$ & $0,90 \pm 0,13$ & $0,12 \pm 0,01$ \\
\hline 13 & Zrigat & Errachidia & $34,1 \pm 3,0$ & $2,08 \pm 0,12$ & & & \\
\hline 14 & Erfoud & Tafilalt & $64,1 \pm 7,5$ & $2,64 \pm 0,21$ & & & \\
\hline 15 & Rissani & Tafilait & $54,7 \pm 4,8$ & $2,26 \pm 0,11$ & & & \\
\hline \multicolumn{8}{|c|}{ Sources thermales } \\
\hline 16 & Sidi Harazem ${ }^{1}$ (s) & Fès & $6,1 \pm 0,7$ & $6,45 \pm 0,53$ & $51,9 \pm 1,3$ & $0,175 \pm 0,02$ & $8,58 \pm 1,04$ \\
\hline 17 & Sidi Harazem ${ }^{2}(s)$ & Fès & $5,2 \pm 0,6$ & $7,39 \pm 0,62$ & $60,4 \pm 1,6$ & $0,189 \pm 0,02$ & $11,59 \pm 1,39$ \\
\hline 18 & Sidi Harazem (c) & Fès & $5,2 \pm 0,5$ & $6,94 \pm 0,56$ & $89,1 \pm 1,2$ & $0,182 \pm 0,01$ & $17,17 \pm 1,73$ \\
\hline 19 & Ain Allah ${ }^{1}$ & Fès & $5,4 \pm 0,6$ & $4,76 \pm 0,32$ & $30,0 \pm 0,0$ & $0,103 \pm 0,004$ & $5,56 \pm 0,61$ \\
\hline
\end{tabular}


Tableau 1 (suite) Activités et rapports d'activité des isotopes de l'uranium et du radium dans les échantillons analysés d'eau de puits et de sources thermales.

Table 1 (continued) Activities and activity ratios of uranium and radium radio-isotopes in analyzed well and thermal spring water samples.

\begin{tabular}{|c|c|c|c|c|c|c|c|}
\hline$n^{\circ}$ & échantillon & région & ${ }^{238} \mathrm{U}(\mathrm{mBq} / \mathrm{l})$ & ${ }^{234} \mathrm{U} /{ }^{238} \mathrm{U}$ & ${ }^{226} \mathrm{Ra}(\mathrm{mBq} / \mathrm{l})$ & ${ }^{228} \mathrm{Ra} /{ }^{226} \mathrm{Ra}$ & ${ }^{226} \mathrm{Ra} /{ }^{238} \mathrm{U}$ \\
\hline \multicolumn{8}{|c|}{ Sources thermales } \\
\hline 20 & Aïn Allah² & Fès & $8,5 \pm 1,0$ & $3,13 \pm 0,26$ & $26,8 \pm 0,6$ & $0,09 \pm 0,01$ & $3,15 \pm 0,39$ \\
\hline 21 & My Yacoub & Fès & $6,5 \pm 0,8$ & $1,84 \pm 0,25$ & $3696,0 \pm 12,0$ & $0,168 \pm 0,003$ & $573 \pm 66$ \\
\hline 22 & Oulmès (c) & Oulmès & $0,6 \pm 0,1$ & $4,13 \pm 0,74$ & $1248,0 \pm 3,0$ & $0,053 \pm 0,001$ & $2115 \pm 321$ \\
\hline 23 & Sidi Ali (c) & Oulmès & $5,1 \pm 0,8$ & $0,97 \pm 0,15$ & $9,1 \pm 0,6$ & $0,06 \pm 0,01$ & $1,79 \pm 0,29$ \\
\hline 24 & Ouled Reguia & F.B. Salah & $17,9 \pm 1,2$ & $2,30 \pm 0,12$ & $122,0 \pm 2,1$ & $0,08 \pm 0,01$ & $6,82 \pm 0,46$ \\
\hline 25 & My Hachem & Errachidia & $13,7 \pm 1,8$ & $3,60 \pm 0,48$ & & & \\
\hline 26 & My Ali & Errachidia & $3,0 \pm 0,3$ & $2,86 \pm 0,31$ & & & \\
\hline 27 & Bleu Meski & Errachidia & $15,6 \pm 1,9$ & $3,03 \pm 0,35$ & & & \\
\hline
\end{tabular}

(s) : prélevé de la source (c) : eau minérale commercialisée ${ }^{1}: 1^{\text {er }}$ échantillonnage ${ }^{2}: 2^{\mathrm{e}}$ échantillonnage. 
d'après plusieurs auteurs (CHERDYNSTEV (1971), IVANOVITCH et HARMON (1992)). De nombreux facteurs sont à l'origine de ce fractionnement isotopique et plusieurs théories concernant ce déséquilibre ont été élaborées (RHOSOLT et al. (1963), KIGOSHI (1971), FLEISCHER et RAABE (1978), FLEISCHER (1980), ANDREWS et KAY (1983)). Des processus de différentiation physiques et chimiques tels que l'effet de recul des atomes et l'effet d'oxydation peuvent produire ce déséquilibre.

Les rapports d'activité ${ }^{234} \mathrm{U} /{ }^{238} \mathrm{U}$ mesurés varient entre 0,79 et 2,83 avec une valeur moyenne de 1,47 dans l'eau de puits et entre 0,97 et 7,39 avec une valeur moyenne de 3,95 dans l'eau de sources. Cette tendance inverse a été, également, signalée par ASIKAINEN et KAHOLS (1979) et SZABO (1982) dans des milieux comparables.

Les activités et les rapports d'activité mesurés dans les échantillons d'eau de puits, d'eau minérale ou dans les échantillons d'eau de table, sont de même ordre de grandeur que ceux trouvés dans d'autres régions du monde (IVANOVITCH et HARMON (1992), REMY et LEMAITRE, (1990)). Les activités mesurées sont très inférieures aux limites maximales admissibles retenues pour l'eau de boisson qui sont, d'après REMY et LEMAITRE (1990): $62,5 \mathrm{~Bq} / \mathrm{l}$ pour ${ }^{238} \mathrm{U}, 50$ $\mathrm{Bq} / \mathrm{l}$ pour ${ }^{234} \mathrm{U}, 8,75 \mathrm{~Bq} / \mathrm{l}$ pour ${ }^{226} \mathrm{Ra}$ et $11,25 \mathrm{~Bq} / \mathrm{l}$ pour ${ }^{228} \mathrm{Ra}$. Ces limites ont été calculées à partir des limites annuelles d'incorporation $(\llcorner\mathrm{Al})$ recommandées par la Commission Internationale de Protection Radiologique (CIPR) et reprises dans la réglementation francaise (Brochure JORF, 1990). Les activités du ${ }^{226} \mathrm{Ra}$ et $\mathrm{du}{ }^{228} \mathrm{Ra}$ et le rapport ${ }^{226} \mathrm{Ra} /{ }^{238} \mathrm{U}$ relativement très élevés pour l'échantillon d'eau de la source My. Yacoub par rapport aux autres échantillons analysées, sont comparables à ceux rapportés par DORETTI et al. (1992), où les activités en ${ }^{226} \mathrm{Ra}$ des eaux utilisées en thérapie thermales en Abano Terme (Italie) ont été trouvées comprises entre 300 et $6400 \mathrm{mBq} / \mathrm{l}$.

Les résultats donnés par les échantillons d'eau de la source Sidi Harazem, qu'ils soient prélevés directement de la source, ou pris de bouteilles commercialisées après avoir subi un traitement, sont pratiquement identiques sauf pour le ${ }^{226} \mathrm{Ra}$ dont l'activité est légèrement plus élevée dans l'eau commercialisée.

\section{5 - CONCLUSION}

L'analyse radioactive de 15 échantillons d'eau de puits et de 12 échantillons d'eau de sources thermales, nous a permis d'avoir une idée générale de la répartition de l'activité des principaux isotopes de l'uranium et du radium dans ces 2 sources d'eau potable au Maroc. Les résultats obtenus permettront d'orienter les prochaines compagnes d'échantillonnage et contribueront à établir une carte de la répartition de la radioactivité naturelle dans les différentes sources d'eau naturelle au Maroc. Ils permettront également de combler le vide qui existe en ce qui concerne les données sur la radioactivité dans les eaux souterraines et les eaux de surface d'une manière générale en Afrique et dans le Monde Arabe. Les activités mesurées sont comparables à celles déjà trouvées dans d'autres régions du Monde. 
À côté des eaux prélevées directement des sources, des eaux minérales commercialisées, dites de table et ayant subi un traitement avant l'embouteillage, présentent les mêmes caractéristiques de radioactivité que les premières.

II ressort de cette étude que la radioactivité des eaux potables analysées est très faible et que les risques sanitaires liés à la consommation de ces eaux sont négligeables.

\section{RÉFÉRENCES BIBLIOGRAPHIQUES}

ANDREWS J.N., KAY R.L., 1983. The U content and ${ }^{234} \mathrm{U} /{ }^{238} \mathrm{U}$ activity ratio of dissolved uranium in groundwater from some triassic sandstones in England. Isot. Geosci., 1, 101-117.

ASIKAINEN M., KAHOLS H., 1979. Anomalously high concentrations of uranium, radium and radon in water from drilled wells in the Helsinki region. Geochim. Cosmochim. Acta, 43, 1681-1686.

Brochure JORF $n^{\circ} 1420,1990$. Décret du 18 avril 1988.

CHERDYNTSEV V.V., 1971. Uranium-234. Israel Program for Scientific Translations, Jerusalem.

CHOUKRI A., REYSS J.-L., TURPIN L., BERRADA M., 1994. Radiochemical separation of $U$ and $T h$ isotopes in phosphate rich samples for age determination. Radiochim. Acta, 65, 137-139.

DORETTI L.D., GEBASI R.G., BATTISTON G., 1992. Natural radionuclides in the Muds and waters in thermal therapy in Abano Terme. Italy. Radiat. Protect. Dosim., 45, 1/4, 175-178.

FLEISCHER R.L., RAABE O.G., 1978. Recoiling alpha-emitting nuclei. Mechanisms for uranium series disequilibrium. Geochim. Cosmochim. Acta, 42, 973-978.

FLEISCHER R.L., 1980. Isotope disequilibrium of uranium, alpha-recoil damage and preferential solutions effects. Science, 207, 979-981.

FRANZ S., 1989. Determination of Radon222 and Ra-226 in Mineral water and drinking water. A survey in Austria. Analyst, 144, 1345-1347.

IVANOVICH M., HARMON R.S., 1992. Uranium series disequilibrium: Applications to earth, marine and environmental sciences $\left(2^{\text {nd }}\right.$ edition). Cladendon Press, Oxford, $910 \mathrm{p}$.

KIGOSHI, K., 1971. Alpha-recoil thorium234. Dissolution into water and the uranium-234/uranium-238 disequilibrium in nature. Science, 173, 47-48.

REMY M.L., LEMAITRE N., 1990. Eaux minérales et radioactivité. Hydrogéologie, 4, 267-278.

RHOSOLT J.N., SHIELDS W.R., GARNER E.L., 1963. Isotopic fractionation of uranium in sandstone. Science, 139, 224226.

SCHMIDT S., REYSS J.-L., 1996. Radium as internal tracer of Mediterranean Outflow Water. Journ. Geophys. Res., 101, 35893596.

SZABO B.J., 1982. Extreme fractionation of ${ }^{234} \mathrm{U} /{ }^{238} \mathrm{U}$ and ${ }^{230} \mathrm{Th} /{ }^{234} \mathrm{U}$ in spring waters, sediments, and fossils at Pomme de Terre Valley, southwestern Missouri. Geochimica et Cosmochimica Act, 46, 16751679.

TOULHOAT M., PHOLLIGER P., MÉNÈS J., 1988. Analyses of lead isotopes and Useries disequilibrium in groundwaters and possible source rocks in the west Morvan area (France). Uranium, 4, 307325. 